\title{
1 A mixed model approach for estimating 2 drivers of microbiota community 3 composition and differential taxonomic 4 abundance
}

5 Amy R Sweeny ${ }^{1 *}$, Hannah E Lemon ${ }^{1 *}$, Anan Ibrahim ${ }^{1}$, Kathryn A. Watt ${ }^{1}$, Kenneth

6 Wilson ${ }^{3}$, Dylan Z Childs ${ }^{4}$, Daniel H Nussey ${ }^{1}$, Andrew Free ${ }^{2 \$}$, Luke McNally ${ }^{1 \$}$

$8 *$ These authors contributed equally

9 \$ Joint last authors

10 Affiliations

$11{ }^{1}$ Institute of Evolutionary Biology, University of Edinburgh, Edinburgh EH9 3FL UK

$12{ }^{2}$ Institute of Quantitative Biology, Biochemistry and Biotechnology, University of Edinburgh

13 EH9 3FL UK

$14{ }^{3}$ Lancaster Environment Centre, Lancaster University, Lancaster, LA1 4YQ, UK

$15 \quad{ }^{4}$ Department of Animal \& Plant Sciences, University of Sheffield, Sheffield, UK

17 Corresponding author: Amy R. Sweeny, amyr.sweeny@gmail.com

Running title: GLMM approaches to metabarcoding data

21 Key words: microbiota, metabarcoding, 16S, amplicon sequence variants, generalised linear

22 mixed effects model, community composition, differential abundance, Bayesian estimation 


\section{Abstract}

25 1. Next-generation sequencing (NGS) and meta-barcoding approaches have revolutionized understanding of within-host communities, such as the gut microbiome, in humans and laboratory animals. The application of such approaches in wild animal populations is growing, but there is a disconnect between the widely-applied generalised linear mixed model (GLMM) approaches commonly used to study phenotypic variation and the statistical toolkit from community ecology which is typically applied to meta-barcoding data.

2. Here, we describe and illustrate a novel GLMM-based approach for analysing the taxonspecific sequence read counts derived from standard meta-barcoding data. This approach allows us to decompose the contribution of different drivers of variation in community structure (e.g. year, season, individual host), via interaction terms in the random effects structure of the model. We also show how these models can be used to determine the degree to which specific taxa or taxonomic groups are responsible for variance attributed to different drivers.

3. To illustrate this approach, we applied it to two cross-sectional meta-barcoding data sets from the Soay Sheep population of St. Kilda. The GLMM approach yielded results that were in agreement with more classical approaches from community ecology, showing that variation the gut microbiota community in these sheep was better explained by age group than by season. We were able to quantify the contributions of different sources of variation to community structure, and also to drill down into the model predictions to show that the age effects we observed were principally due to increases in taxa of the phyla Bacteroidetes and declines in taxa of the phyla Firmicutes.

4. Our proposed models offer a powerful new approach to understanding the drivers of variation in estimates of community structure derived from meta-barcoding data. We discuss how our approach could be readily adapted to allow researchers to estimate that contribution of host genotype, environment, and microbial/parasite phylogeny to observed community structure, and thus provide a powerful means to answer emerging questions surrounding the ecological and evolutionary roles of within-host communities. 


\section{1. Introduction}

55 The ecological dynamics of within-host communities of parasites and commensal microbes can 56 have dramatic effects on host health and fitness (Alberdi et al. 2016; Koskella et al. 2017). One

57 increasingly well studied example of such a within-host community is the so-called gut

58 microbiota: the often complex and diverse community of commensal bacteria resident to the

59 gastrointestinal tracts of their animal hosts. As well as playing a crucial role in the digestion of

60 food, studies from humans and model laboratory animals highlight impacts of the gut microbiota

61 on host behaviour, metabolism, as well as endocrine and immune homeostasis (Sudo et al. 2004;

62 Cox-Foster et al. 2007; Desbonnet et al. 2014; Wahlström et al. 2016). A growing number of

63 studies within ecology and evolutionary biology are seeking to study the dynamics of the gut

64 microbiota of natural systems using a combination of faecal sampling and next-generation

65 sequencing (NGS) meta-barcoding approaches. Understanding the role of within-host

66 communities in underpinning host phenotypic variation, as well as wider ecological and

67 evolutionary dynamics, in the wild will require statistical approaches that allow us to robustly

68 quantify the contribution of different environmental and host-related factors to such meta-

69 barcoding data. Generalised linear mixed models (GLMM) are a well-established and widely

70 used suite of statistical models within ecology and evolution which provide a flexible means for

71 appropriately dealing with the complex data structures and relationships between predictors of

72 interest that arise in ecological data (Bolker et al. 2009). Although they have yet to be widely

73 applied in this context, GLMMs have huge potential to help dissect and understand the drivers of

74 within-host community dynamics, as revealed by meta-barcoding data.

76 Standard methodologies for investigating hypotheses concerning gut microbiota dynamics in the

77 wild typically include collection of faecal samples from selected study subjects and application

78 of next-generation sequencing (NGS) techniques for meta-barcoding of informative bacterial

79 genes for taxonomic assignment of sequenced reads ( $\mathrm{J}$ et al. 2018). Microbiota community

80 analysis commonly relies on transformation of OTU (operational taxonomic units) or ASV

81 (amplicon sequence variant) counts into relative proportions per sample or rarefaction such that a

82 set library size is randomly subsampled from all samples (McMurdie \& Holmes 2014; Weiss et

83 al. 2017; McMurdie 2018) Hypothesis testing using transformed counts from 16S taxonomic

84 assignments typically is focused on community-level differences in taxonomic diversity and 
85 composition between experimental groups or time points of interest. Statistical approaches to this

86 end include estimation of alpha diversity (the number of distinguishable taxa within a sample),

87 distance measures (e.g. Bray-Curtis dissimilarity (Bray and Curtis, 1957)), and ordination with

88 dimensionality reduction (e.g. principal coordinates analysis). Data transformations and

89 hypothesis tests in these approaches have several limitations. Standardisations of data based on

90 proportions ignore heteroscedasticity from different library sizes across samples, while those

91 relying on rarefaction restrict data such that the reads considered per each sample are limited to

92 the minimum number of reads across all samples (McMurdie \& Holmes 2014). This in turn can

93 significantly elevate rates of false positives or reduce performance in microbiome clustering

94 approaches. In addition to statistical pitfalls, these traditional approaches for assessing

95 community-level differences differ philosophically from GLMM-based approaches which

96 partition complex sources of variance. Although traditional approaches have provided substantial

97 insights into microbiota community composition, they fall short of the flexibility and power

98 offered by GLMM-based approaches to dissect the manifold and complex contributors to

99 variation in measured phenotypes in natural populations. There has therefore been movement

100 among community ecologists toward such model-based methods (Niku et al. 2019). Here we

101 develop a GLMM-based approach to decompose the sources of variation in count data derived

102 from meta-barcoding approaches and discuss the advantages of this approach for analysis of

103 microbiota and other community data.

105 The application of mixed effects models to microbiota datasets is not new. The "Hierarchical

106 Modelling of Species Communities" (HMSC) approach uses latent variable modelling and

107 random effects to model community compositions 1 and has been applied to the composition of

108 the microbiota (refs). A similar approach developed for microbiota datasets has also shown

109 insights into microbiota composition in the wild (ref Bjork). Our suggested approach differs from

110 these in several ways. Firstly, these approaches have a focus on modelling correlations among

111 microbial taxa using latent variables to model residual correlation, which adds quite a lot of

112 complexity to the modelling process. Our approach does not attempt to model these correlations,

113 it focuses on variance decomposition of the sort familiar to ecologists and evolutionary biologists

114 working on wild systems. If correlations among microbial taxa is of primary interest we would

115 direct readers to these approaches. Secondly, our approach does not require the use of any

116 particular modelling package or a high degree of proficiency in coding. There are two central 
117 ideas in our approach - using sample level random effects in Poisson models to account for

118 variability in library size, and using random effects of microbial taxonomy to allow for effects of

119 host and environment on microbiota composition - that can be implemented in almost any

120 random effects modelling software or packages with which the reader is familiar. Thus, at the

121 expense of modelling residual correlation among species, our approach offers a familiar method

122 to decompose sources of variance in the microbiota for field scientists. Below we outline the

123 motivation for this approach and illustrate this via an application to two 16S metabarcoding

124 datasets from a wild mammal.

\section{2. A GLMM approach}

127 As gut microbes have such important effects on host physiology, behaviour and health much

128 research has sought to identify individual microbial taxa that are responsible for alterations of

129 host phenotype and state. This has in many ways mirrored the goals of many genome-wide

130 association study (GWAS) analyses, which have sought to identify particular genetic variants

131 associated with phenotypes of interest, often with a goal of developing diagnostics or drug

132 targets (Visscher et al. 2012). However, just as GWAS analyses have shown us that most

133 phenotypes are highly polygenic, being determined by a complex combination of genetic

134 variants of small effects (Goldstein 2009; Consortium 2009; Loh et al. 2015), the study of host-

135 associated microbiomes has often failed to find single taxa associated with host states (Clemente

136 et al. 2012; Vayssier-Taussat 2014). Instead many changes in host state are associated with

137 general shifts in microbiome composition, often termed dysbiosis (Clemente et al. 2012; Carding

138 et al. 2015). Phenomena such as dysbiosis shift the level at which we look for associations with

139 host phenotype move from a small number of microbial taxa to the whole microbiota. In

140 addition, the most pressing questions about host-associated microbiota in ecology and evolution

141 are very general and focused on the entire microbiota community (Koskella et al. 2017). For

142 example, what are the relative roles of host physiology and environment in shaping the

143 microbiota? How heritable is the microbiota? How much does microbiota composition impact

144 fitness? The shift in focus of these questions from individual taxa to complete community poses

145 an important conceptual and statistical challenge. 
147 As previously discussed, host-associated microbiotas often constitute hundreds or thousands of 148 different taxa. Whenever we need to estimate a large ensemble of related parameters, a common 149 statistical approach is to treat them as random variables from some distribution (Gelman \& Hill 150 2006). To understand how this approach applies to the microbiota let us consider the concrete 151 question of estimating how the composition of the gut microbiota might change with season in a 152 wild mammal. In traditional approaches to analysing microbiota datasets, it would be common to 153 visualise an ordination of the data, distinguishing points by season. Then, one would perform a 154 permutational ANOVA on a dissimilarity matrix to test if microbiotas from different seasons are 155 more dissimilar than those from the same season, and go on to test for differential abundance of 156 individual taxa across seasons to identify taxa with a major role in these changes (Segata et al.

157 2011; Mandal et al. 2015). In this approach, estimates for how individual taxa differ by season

158 are all independent of each other. Using a random effects model would approach this in a 159 fundamentally different manner, where a model would be fitted with a random effect for the 160 seasonal change for each microbial taxon. This approach has the advantage that all taxa inform 161 the estimate of the mean and variance of the distribution that the effects across taxa come from.

162 The estimates of parameters for individual taxa are then "shrunk" to this distribution. This

163 "shrinkage" is known to improve the accuracy of parameter estimation as long as there are large 164 numbers of groups for the random effects, which is generally true for most host-associated 165 microbiota owing to their large number of taxa.

167 While fitting such random effects models is known to improve parameter estimation owing to 168 shrinkage, its biggest advantage is in allowing us to shift the questions we ask to the whole 169 microbiota level, and partition complex \& inter-related sources of variance. GLMM approaches 170 have been used across other ecological and evolutionary contexts to estimate repeatability, 171 relative levels of spatiotemporal variance (Albery et al. 2019), social and common environment 172 effects (Rushmore et al. 2013; Froy et al. 2018), as well as heritability and the role of host 173 genetics (Hayward et al. 2014). Answering such questions has proved hugely challenging in the 174 microbiota field as most analyses rely on tools which are not multi-level, from which it is 175 extremely difficult to decompose the relative contribution of simultaneous processes at the host 176 and environmental scale. However, mutli-level models have been shown to offer significant 177 advantages over many other compositional methods in community ecology for species 178 abundance data (Jackson et al. 2012). Here, we develop and illustrate on method to appropriately 
179 structure random effects across microbial taxa within a community using a GLMM, and thus

180 partition the sources of variance driving microbiota composition.

182 To see how such a model can be structured let us again return to the example of estimating the

183 effect of season on the gut microbiota of a wild mammal (Figure 1). Consider a scenario with

184 two samples taken per host from a sample of hosts in a population, one in winter and one in

185 summer, and with samples appropriately sequenced and reads bioinformatically assigned to

186 ASVs. This will yield data in the form of a count of reads belonging to each ASV (the focal

187 taxonomic group) within each sample (Figure 1A), with two samples per individual host one

188 from each season. We can directly analyse such count data by fitting a Poisson family GLMM

189 with log-link. The predicted values on the link scale are given according to the following model

190 (Figure 1B).

$$
\log \left(y_{h, a s v, s}\right)=\beta_{0}+\beta_{1} s+u_{h}+u_{a s v}+u_{h: s}+u_{a s v: h}+u_{a s v: s}+u_{a s v: h: s}
$$

194 Here $y_{h, a s v, s}$ is the read count, $\beta_{0}$ is a global intercept, and remaining terms account for technical

195 variation effects in read counts (abundance) as well as biological variation in taxonomic

196 composition. Fixed and random terms dealing with technical variation are as follows: $\beta_{1}$ is the

197 effect of season $(s)$ on total read count, $\mathrm{u}_{\mathrm{h}}$ is a random effect describing variation in total read

198 count among individual hosts (h, where there are multiple samples per host), $u_{a s v}$ is a random

199 effect describing variation in total read count of each ASV across samples and hosts, $\mathrm{u}_{\mathrm{h}: \mathrm{s}}$ is a

200 random effect accounting for library size by describing variation in mean read count in each

201 sample (i.e. host by season), and $u_{a s v: h: s}$ is an additional random effect accounting for row-level

202 variation (over-dispersion). In this example biological effects of interest are specified as follows:

$203 u_{a s v: h}$ is a random effect describing the abundance (read count) of an ASV in host $h, u_{a s v: s}$ is a

204 random effect describing how ASV abundances (read counts) ASV differ between seasons. By

205 apportioning the variance attributed to these different random effects we can assess the relative

206 contributions of these different factors to microbiota composition (Figure 1B-C). For example, a

207 high variance associated with $u_{h: s}$ would indicate a high degree of technical variation due to

208 library size variation across samples, and high variance associated with $u_{\text {asv }}$ could indicate

209 variation in read counts across ASVs due to over-dispersion introduced by many rare taxa

210 (Figure 1C). With regard to biological inference, variance associated with $u_{a s v: h}$ can be 
211 interpreted as indicative of individual 'repeatability' of ASV community composition and $u_{a s v: s}$

212 can be interpreted as reflecting variance associated with compositional shifts across seasons.

214 Continuing with the above example, we can further use Poisson model outputs to explore which

215 specific taxa are driving differential abundances between groups of interests (e.g. season), which

216 is commonly of great interest in microbiome studies, but for which many existing methods may

217 be affected by library size and normalisation methods (Weiss et al. 2015). Differential

218 abundance in this example can be estimated using each ASV-by- season level of the random

219 effect $u_{a s v: s}$ and comparing posterior distributions for each ASV across factor levels. For

220 example, the mean of the posterior distribution for ASV1:summer - that for ASV1:spring can be

221 interpreted as the differential abundance of ASV1 between spring and summer, allowing

222 identification of ASVs which exhibit the largest deviations from the means.

224 While the Poisson model accounts for variation in library size across samples, there has also

225 been a shift in microbiota research towards explicitly compositional data analysis, which

226 removes any effects of library size (other than in quantifying uncertainty) prior to analysis. The

227 centered log-ratio (CLR) described by Aitchsion (1982) represents one such transformation. We

228 present full details of how to implement the above GLMM approach using CLRs, and then apply

229 this alternative parameterization to the example data described below, in our Supplementary

230 Files. 

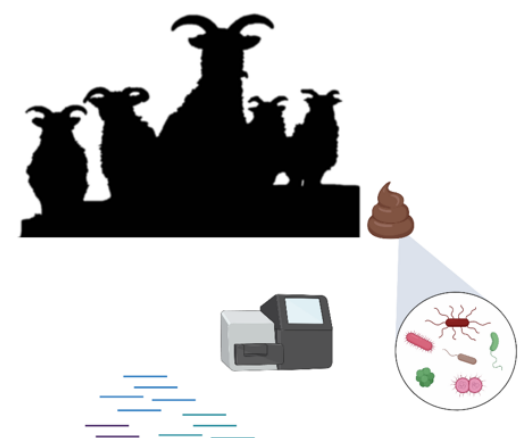

Feature

abundances per

A

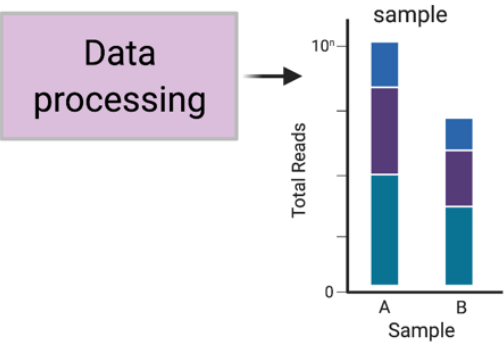

Wild Microbiota Sampling Characteristics
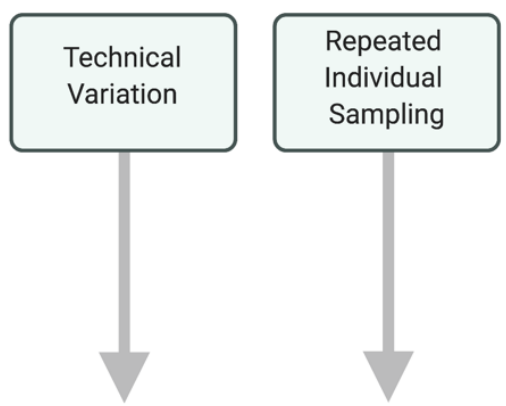

Host \&

Environment

Variation

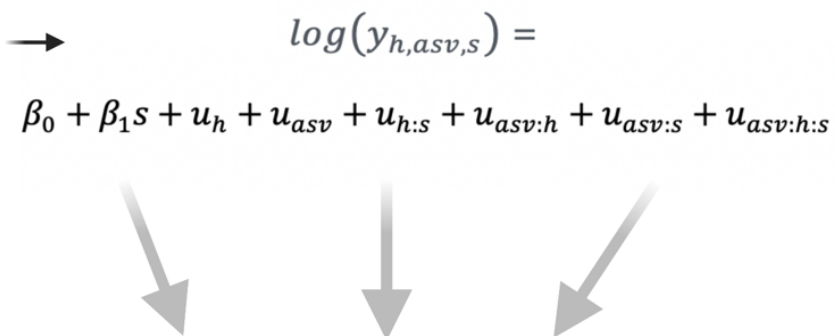

$u_{h}+u_{a s v}+u_{h: s}+u_{a s v: h}+u_{a s v: s}+u_{a s v: h: s}$

B

Mixed Model

Approach

C

Inference
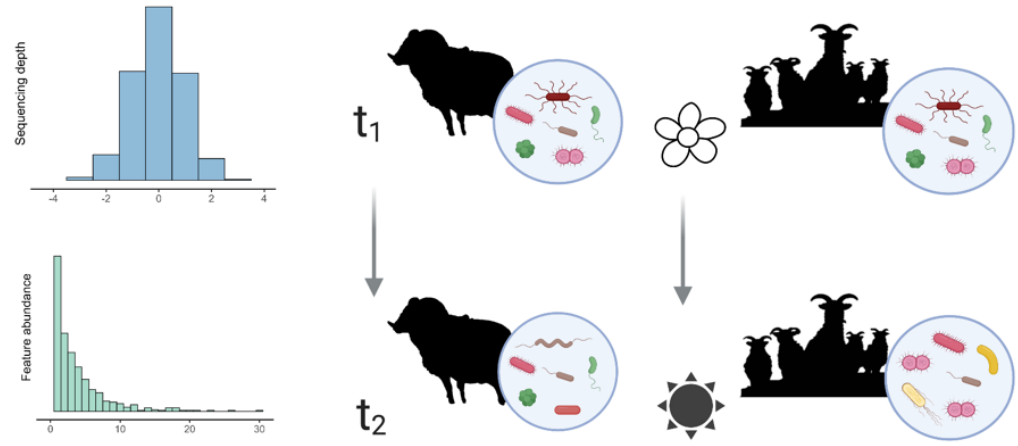

Technical variation Abundance
Individual repeatability

Seasonal effects 
Figure 1. Overview of mixed model approach to wild microbiota analysis. Data processing (A) generates amplicon sequence variant (ASV)-level abundances for each sample. These raw abundances are used as the response for generalised linear mixed-effects models with Poisson error families. In the example illustrated, data includes sampling timepoints for a group of individuals taken during two seasons. Model syntax therefore specifies a fixed effect of age, and random effects for taxonomy (asv), sample id (host:season, h:s), individual differential abundance of asvs (asv:h), differential abundance of asvs across seasons (asv:s), and a residual variance at the row-level (asv:h:s). GLMM output can be used to partition the variance explained by each random effect term (B). These variance components can be interpreted as the relative contributions of both technical variation and host or environmental contributions to differential abundance as illustrated in (C). Created with BioRender.com

\section{A worked example: age and season effects on gut} microbiota in wild sheep

247 To test and illustrate our approach, we obtained faecal samples from Soay Sheep (Ovis aries)

248 from the island of Hirta in the St. Kilda archipelago of the Outer Hebrides of Scotland. These

249 animals are free-living and are part of a long-term study in which individuals have been marked

250 and monitored longitudinally since 1985 (Clutton-Brock Pemberton 2004). All animals sampled

251 had been caught and uniquely tagged within a few days of birth, so that their age and sex were

252 known with certainty. Each year, fieldwork teams visit St Kilda in spring to monitor lambing and

253 capture, mark and sample newborn lambs within a few days of birth. Subsequently, each August

254 a larger field team visits to capture, mark and sample animals living in the study area using a

255 series of corral traps (Clutton-Brock \& Pemberton 2004).

Two sets of faecal samples were collected, in 2013 and 2016, to allow comparison of the gut

258 microbiota of individuals of different ages (2013) and from the same individuals sampled in

259 different season (2016). The 2013 samples were collected during the August catch and included

26030 samples from lambs (around 4 months old) and 28 samples from older adults (ages 2-13). The

2612016 samples were collected from a set of 36 females age 1-13 years who were sampled in both

262 spring (around the time of parturition) and then 3-4 months later in August. Microbial DNA was

263 extracted from samples, amplified using bacterial 16S rRNA V4 region primers, and sequenced

264 using the Ilumina MiSeq platform to generate 250 base pair (bp) paired-end reads. Sequences

265 were processed using the DADA2 pipeline in R (v1.12.1) to call amplicon sequence variants

266 (ASVs) (Callahan et al. 2016). Full details of sampling, sequencing, and data processing

267 methods are provided in the Electronic Supplementary Material (ESM 1.1-1.4). We conducted 
268 standard dissimilarity analysis and PERMANOVA tests on effects of age and season for

269 comparison to the results of our GLMM approach (see ESM 1.4.1 for full details).

\subsection{Specification of GLMMs:}

272 We applied separate GLMMs to the 2013 and 2016 datasets. First, an aggregate dataset for each

273 year was created from the sample metadata, taxonomic classifications for each ASV, and an

274 ASV-by-sample abundance matrix. We fit GLMMS with Poisson errors and log links to each

275 dataset using the Bayesian package 'MCMCglmm' (Hadfield 2010) using the approach

276 introduced in Section 2. Models fit to 2013 data, which included samples from hosts of two age

277 classes from a single season (one sample per host) were specified as follows:

$$
\log \left(\bar{y}_{h, a s v}\right)=\beta_{0}+a \beta_{a}+u_{h}+u_{a s v}+u_{a s v: a}+u_{a s v: h}
$$

Where $y_{h, a s v}$ is the read count per ASV within each host, $\beta_{0}$ is a global intercept, $\beta_{a}$ is the effect of age ( $a$, binary factor: lamb versus adult) on total read count, $\mathrm{u}_{\mathrm{h}}$ is a random effect random effect describing variation in total read count among individual hosts (h, equivalent to sample here where hosts are sampled once each), $u_{a s v}$ is a random effect describing variation in total read count of each ASV across hosts/samples, $u_{a s v: a}$ is a random effect a random effect describing how ASV abundances (read counts) ASV differ between host age classes and $u_{a s v: h}$ is an additional is an additional row-level random effect describing residual variation.

Models fit to 2016 data, which included samples from individual hosts of similar age sampled in both spring and summer of the same year (two samples per host), were specified as follows:

$$
\log \left(\bar{y}_{h, a s v, s}\right)=\beta_{0}+s \beta_{s}+u_{h}+u_{a s v}+u_{h: s}+u_{a s v: h}+u_{a s v: s}+u_{a s v: h: s}
$$

294 Here $y_{h, a s v, s}$ is the read count, $\beta_{0}$ is a global intercept, $\beta_{s}$ is the effect of season ( $s$, binary factor:

295 spring versus summer) on total read count, $u_{h}$ is a random effect describing variation in total

296 read count among individual hosts (h, where there are multiple samples per host), $u_{a s v}$ is a

297 random effect by describing variation in total read count of each ASV across samples and

298 hosts, $u_{h: s}$ is a random effect describing variation in total read count among individual hosts (h,

299 where there are multiple samples per host), $u_{a s v: h}$ is a random effect describing the abundance 
300 (read count) of an ASV in host $h, u_{a s v: s}$ is a random effect describing how ASV abundances

301 (read counts) ASV differ between seasons, and $u_{a s v: h: s}$ is an additional row-level random effect

302 describing residual variation.

$304 \beta_{0}$ is a global intercept, and remaining terms account for technical variation as well as biological 305 variation of interest. Fixed and random terms dealing with technical variation are as follows: $\beta_{1}$ 306 is the effect of season $(s)$ on total read count, $\mathrm{u}_{\mathrm{h}}$ is a random effect describing variation in total

307 read count among individual hosts (h, where there are multiple samples per host), $u_{a s v}$ is a

308 random effect by describing variation in total read count of each ASV across samples and hosts

$309, \mathrm{u}_{\mathrm{h}: \mathrm{s}}$ is a random effect accounting for library size by describing variation in mean read count in

310 each sample (i.e. host by season), and $u_{a s v: h: s}$ is an additional random effect accounting for row-

311 level variation (over-dispersion). In this example biological effects of interest are specified as

312 follows: $u_{a s v: h}$ is a random effect describing the abundance (read count) of an ASV in host $h$,

$313 u_{a s v: s}$ is a random effect describing how ASV abundances (read counts) ASV differ between

314 seasons.

316 Using this GLMM approach, we calculated the relative contributions to sources of variance in

317 the data from both technical and biological model components. We followed Nakagawa \&

318 Schielzeth (Nakagawa \& Schielzeth 2013) for calculation of $\mathrm{r}^{2}$ from GLMMs with Poisson error

319 distributions. Using this formula, there is a portion of variance equal to 1 minus the sum variance

320 of the model components which represents variance arising from the Poisson distribution.

321 Where multiple samples are present per individual (2016), repeatability of the community

322 composition of ASVs can be estimated as the proportion of variance attributable to differential

323 taxonomic composition across individuals divided by the sum of the variance explained by all

324 other non-technical component terms estimating compositional effects $u_{a s v: h} /\left(u_{\text {asv:s }}+u_{\text {asv:h }}+\right.$ $\left.325 u_{a s v: h: s}\right)$.

327 We investigated differential abundances as outlined above (2). To extract information of specific

328 bacterial taxa contributing to differential abundance across age groups or season, we used

329 Poisson model outputs and subtracted the posterior distributions for each ASV between group

330 levels (2013: Age; 2016: Season). We used the resultant distribution to calculate a mean

331 difference and highest posterior density interval (HPDI) to estimate differential abundance for 
each ASV. For example, the mean of the posterior distribution for ASV1:summer - that for ASV1:spring can be interpreted as the differential abundance of ASV between spring and summer, where a difference can be considered significant when credible intervals do not span zero. We calculated p-values of each differential abundance by calculating the

336 proportional overlap of each pairwise set of distributions divided by half the number of stored 337 iterations.

\subsection{Results:}

340 The microbiota communities of Soay Sheep were dominated by two phyla, Firmicutes and

341 Bacteroidetes (Figure S1), as has been previous observed in most vertebrates (Ley et al. 2008)

342 Principle coordinates analysis (PCoA) based on Bray-Curtis dissimilarity indicated clustering of

343 samples by age and by season (Figure 2). The result of a PERMANOVA test on the 2013 data

344 set showed a significant difference in group centroids for lambs and adults (pseudo-F $=7.161, \mathrm{p}$

$345<0.001)$, with $11.34 \%$ of the variance in gut microbiota composition $\left(\mathrm{R}^{2}\right)$ is explained by

346 differences between lambs and adults. PERMANOVA results for the 2016 data showed that

347 group centroids for April and August are significantly distinct (pseudo-F = 2.026, $\mathrm{p}=0.002$ ), but 348 season only explains $2.81 \%\left(\mathrm{R}^{2}\right)$ of the observed variance.

Age

Lamb

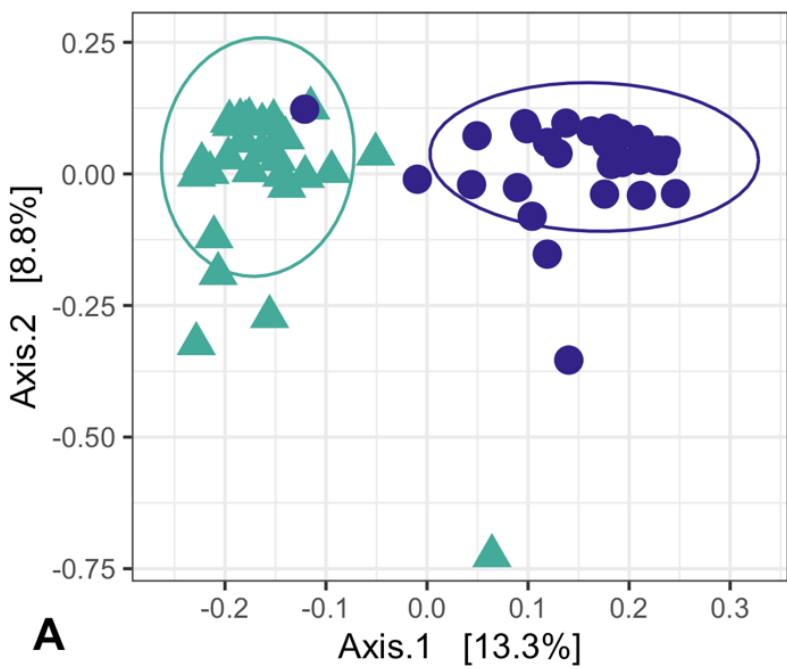

Season Spring Summer

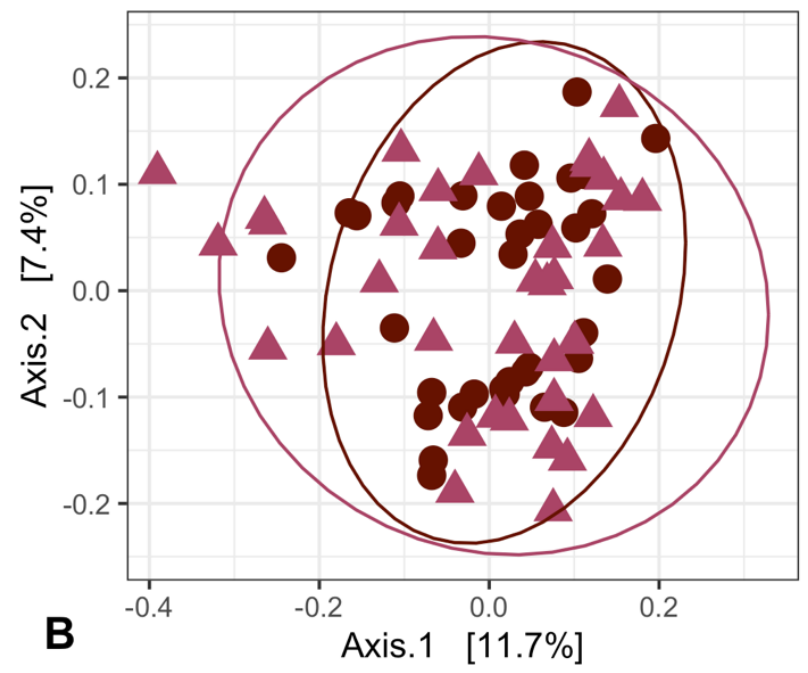

Figure 2. Soay Sheep gut microbiota beta diversity in adults and lambs from 2013 (A) and from April and August of 2016 (B). Principle coordinates (PCoA) plots represent Bray-Curtis dissimilarity indicating clustering of samples by group. Ellipsoids represent a $95 \%$ confidence interval surrounding each group. 
355 Poisson GLMMs from 2013 data showed comparable results to ordination approaches, where 356 community composition differed substantially between age classes (Proportion variance $u_{\text {asv: } a}$ :

357 19.88\% CI 18.47-21.39\%; Figure 3). Additional effects estimated by the model showed a

358 substantial proportion of variance explained by taxonomic variation in ASV abundance $\left(u_{a s v}\right.$ :

$35917.35 \%$ ), a small portion of variation explained by variation in mean library size across samples

$360\left(u_{h} 2013: 1.59 \%\right)$, and considerable residual variance (estimated by the 'units' term in

361 MCMCglmm; $u_{a s v: h}$ 2013: 49.42\%; Figure 3).

363 Poisson GLMMs from 2016 data likewise showed comparable results to ordination approaches, 364 where community composition changed negligibly between seasons $\left(u_{\text {asv:s }}: 1.24 \%\right.$ CI $0.99-$ 365 1.44\%; Figure 3). The 2016 model showed a substantial proportion of variance explained by 366 taxonomic variation in ASV abundance $\left(u_{\text {asv }} 2016: 34.74 \%\right)$, a small portion of variation 367 explained by variation in mean library size across samples $\left(u_{h: s} 2013: 1.99 \%\right)$, and considerable 368 residual variance $\left(u_{a s v: h: s}\right.$ 2013: 46.67\%; Figure 3). Repeated sampling of individuals in 2016 369 additionally showed moderate variance explained by inter-individual variation in community 370 composition $\left(u_{a s v: h} 2016: 5.49 \%\right)$. The equated to an individual repeatability of $11.1 \%$ for their 371 microbiota community composition across sampling time points.

373 As outlined in 3.1 we calculated differential abundances using the saved posterior distributions

374 for each random effect level of specific taxa across age classes (2013 data set; Figure 4A \& B)

375 and seasons (2016 data set; Figure 4C \& D). For the ASV-by-age effect in the 2013 data $\left(u_{a s v: a}\right.$,

376 Figure 3), the estimates of taxa-specific differential abundances suggest that ASVs

377 demonstrating significant shifts between lambs and adults belong primarily to two phyla,

378 Firmicutes and Bacteroidetes (Figure 4A-B). 683 out of 2,023 (33.76\%) ASVs present in 2013

379 data showed significant shifts between lambs and adults (50.81\% positive shifts, $49.19 \%$

380 negative shifts). Bacteroidetes represented 52.16\% of significant positive shifts into adulthood, 381 and Firmicutes represented $72.02 \%$ of the significant negative shifts into adulthood (Figure 4B;

382 Table S4). For the ASV-by-season effect in the 2016 data (2016 $u_{\text {asv:s }}$, Figure 3 \& Table S4), 383 very few ASVs (24 of 2,364; 1.02\%) showed significant differential abundance between spring 384 and summer sampling (Figure 4C-D; Table S4). 
386 Our results indicate that there are substantive developmental shifts in the Soay sheep microbiota

387 between lambs and adults, and that the majority of taxa shifting in abundance belong to the

388 Bacteroidetes and Firmicutes. However, this raises the question of whether this shift is because

389 Bacteroidetes are generally more abundant in adults and Firmicutes more abundant in lambs, or

390 if the ASVs that show these patterns just happen to be in these phyla. To illustrate how GLMMs

391 can be used to address questions of this sort, we modified our models for the 2013 dataset to

392 include additional taxonomic effects of family and phylum, allowing us to identify taxonomic

393 levels most responsible for differential abundances. Details of these phylogenetically more

394 explicit GLMMs and their results and implications are presented in detail in the ESM (see

395 sections 1.4.3, Figure S4, Table S3). Results suggest considerably variation with respect to age

396 across families and ASVs within both the Bacteroidetes and Firmicutes phyla, and that most of

397 the age effects on microbiota community composition occurs at these lower taxonomic levels

398 (Figure S4; Table S3).

A

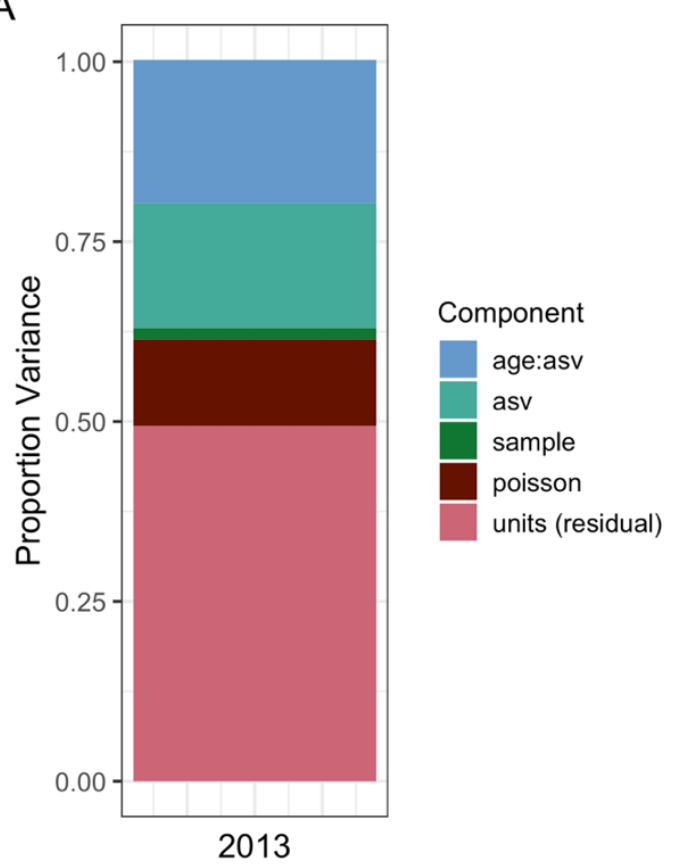

$\mathrm{B}$

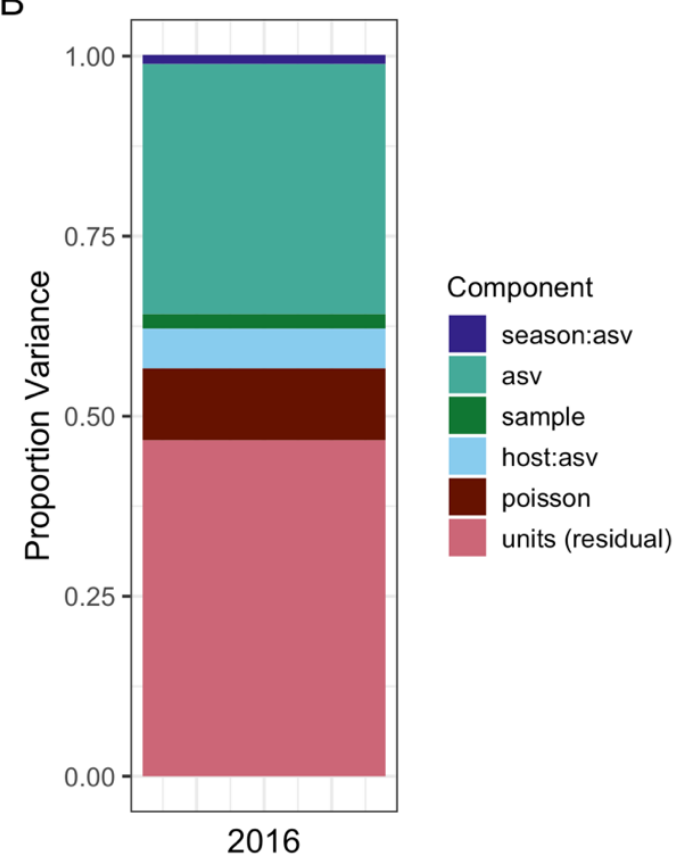

403

404

405

406

407

408 
A

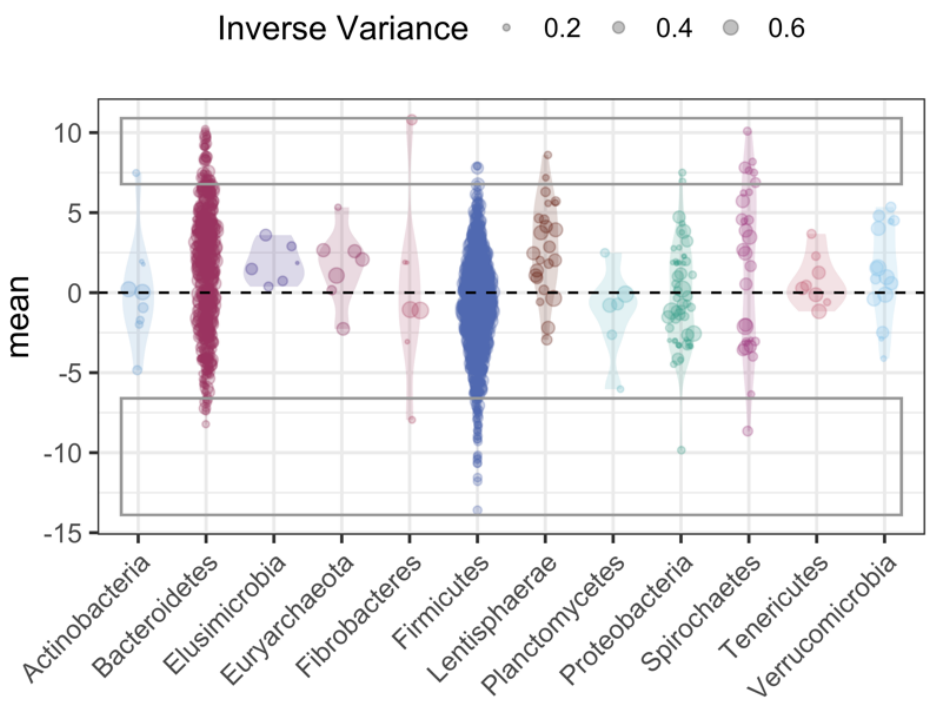

C

Inverse Variance $\cdot 0.8 \cdot 1.0 \cdot 1.2 \circ 1.4 \odot 1.6$

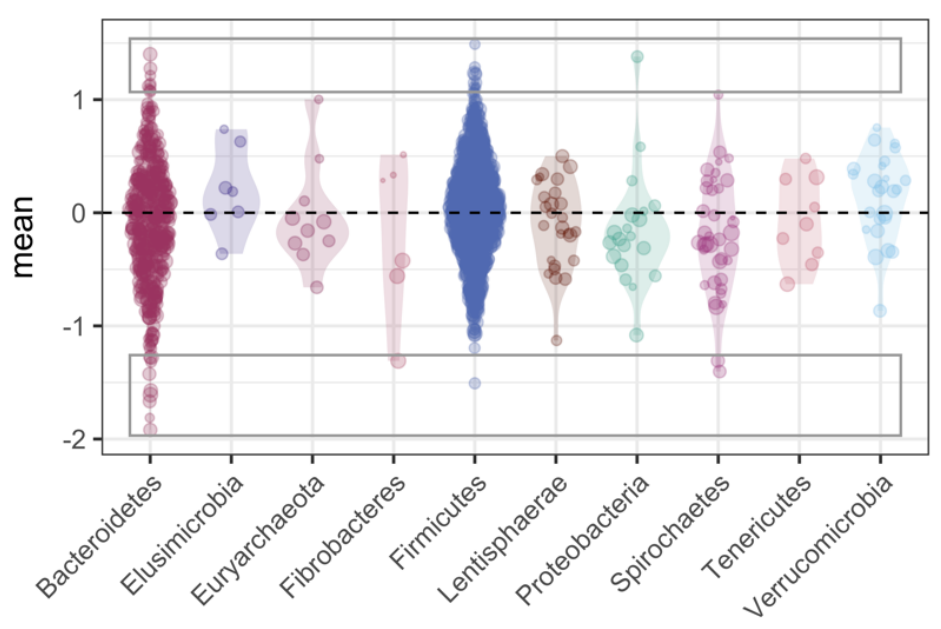

409

410

411

412

413

414

415

416

417

418 decreases of abundance.
B

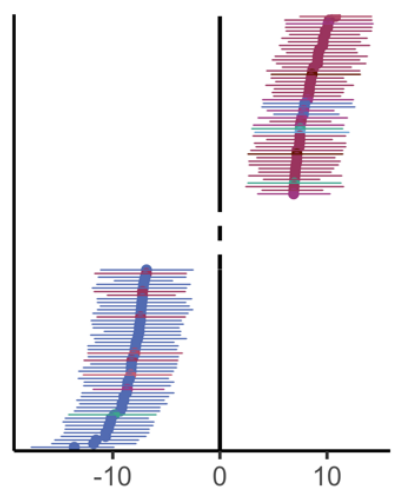

phylum

$\rightarrow$ Actinobacteria

- Bacteroidetes

- Fibrobacteres

- Firmicutes

- Lentisphaerae

- Proteobacteria

- Spirochaetes

- Tenericutes

mean

D

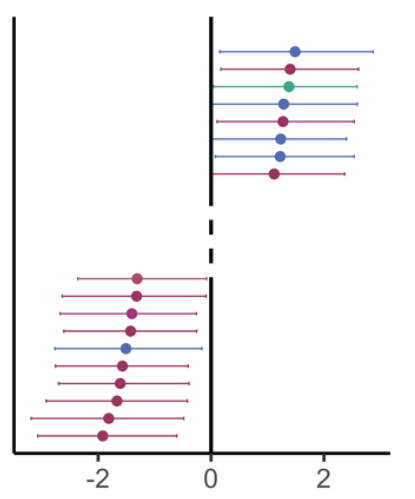

phylum

- Bacteroidetes

- Fibrobacteres

$\rightarrow$ Firmicutes

- Proteobacteria

-- Spirochaetes

mean

Figure 4. Differential abundances across age classes (A-B) or season (C-D) for individual ASVs calculated from GLMMs with Poisson error families and taxonomic level specified as ASV-only. A \& C represent all ASV-level effects. Violin plots represent distribution of effect estimates, size of point represents the inverse variance of the estimate. Rectangles indicate the ASVs with the highest magnitude (positive or negative) differential abundances in Forest plots (B \& D). Forest plots represent point estimate and HPDI for the ASVs involved in the 50 (age class) or 20 (season) strongest increases and 


\section{4. Discussion \& wider applications}

420 We have described a novel approach to analysing meta-taxonomic data derived from NGS using

421 a GLMM framework, and have illustrated this method using data describing variation in the gut

422 microbiota community in wild sheep. Our approach represents an important step forward for

423 researchers interested in using meta-taxonomic approaches to understand variation in community

424 structure in complex, non-experimental settings. It allows the well-established power and

425 flexibility of GLMM-based approaches to be harnessed to decompose drivers of variation in

426 NGS-derived data on the taxonomic composition of samples. Our example analyses provide very

427 simple illustrations of how the approach can be used to estimate the contribution of host-related

428 or environmental factors (specifically, age and season) to variation in the community structure of

429 the gut microbiota. We show that results are comparable to those derived from widely-applied

430 ordination-based approaches, and discuss the implications of observed variation in gut

431 microbiota structure with age and season briefly below. However, these analyses are intended

432 mainly as templates to help illustrate the approach, and barely scratch the surface of the types of

433 important outstanding questions the method could be used to tackle with larger-scale data sets.

434 Applying GLMMs to taxonomic-level sequence count data provides a rich toolkit from fields

435 like quantitative genetics to dissect the contributions of different environmental and host factors

436 to variation in community structure, with the potential to advance our understanding of

437 community ecology, host-microbe or -pathogen interactions, and evolutionary dynamics.

439 In our illustrative analyses of wild Soay sheep, both GLMM and ordination-based approaches

440 identified a stronger effect of age than season on the gut microbiota. Changes in the structure of

441 the gut microbiota across development in early life and during senescence in later adulthood are

442 well-established in human studies (Popkes \& Valenzano 2020), but remain poorly understood in

443 natural populations. Our data clearly show the gut microbiota community structure changes

444 between recently weaned lambs and adults, and argues for further longitudinal studies in natural

445 systems to test whether shifts in gut community structure could play a role in patterns of

446 demographic ageing in the wild. A growing number of studies in the wild have documented

447 seasonal gut microbiota changes (Amato et al. 2015; Maurice et al. 2015; Ren et al. 2017; Orkin

448 et al. 2019). The absence of a strong seasonal different in our data may be related to the relative

449 homogeneity of the herbivorous diet of Soay sheep, as most previous wild studies are of 
450 omnivores with strong seasonal shifts in diet preference. Alternatively, it may be because the 451 spring and summer seasons we sampled in are both periods of relatively high food abundance

452 and quality, and more pronounced change would be observed in autumn and winter when habitat 453 quality and food availability changes more dramatically. In future studies, repeated sampling of

454 the same individuals over time will be crucial to understand the effects of age, environmental and 455 other variables on gut community structure. The application of our approach to such longitudinal 456 metataxonomic data sets will help researchers robustly estimate within-individual patterns of 457 change in community structures over time or space, whilst also estimating how repeatable 458 community structure across hosts.

460 Our novel GLMM approach allows estimation of key ecological and evolutionary parameters 461 from meta-taxonomic data sets which can advance our understanding of within host-microbe 462 evolutionary dynamics. Individual repeatability of measured phenotypes is an important and 463 widely estimated parameter in ecology and quantitative genetics (Wilson 2018). Estimating the 464 within-host repeatability of microbiota community structure over time can offer insight into the 465 extent to which host control and environmental selection determine species composition (Foster 466 et al. 2017). The GLMM structure presented here directly estimates this repeatability across two 467 seasons in our 2016 data set at around 11\%, although the small sample size and temporal 468 proximity of the samples should mean we interpret this parameter estimate with caution.

469 However, the model is illustrative and it should be clear that it is readily extendible to mixed470 effect model of more a more complex nature to address emerging questions in the field. For 471 example, effects of host relatedness \& inbreeding effects on microbiota composition in have 472 been explored previously in microbiome studies but via ordination methods using a small 473 number of genetic clusters as grouping units (Yuan et al. 2015). Including host genetic 474 relatedness matrices as a random effect in a mixed-effects model (so-called "animal models") 475 within our GLMM framework can offer insights into heritability and inbreeding effects on 476 community composition as it compares to other forces in the population (Wilson et al. 2010).

477 Factors which can be of both considerable ecological and evolutionary interest, and that can also 478 confound heritability estimates, such as maternal effects or shared nest or litter effects can 479 likewise be incorporated in these models (Kruuk \& Hadfield 2007). Statistical advances to 480 address maternal and social effects as well spatial autocorrelation in ecological datasets can also 481 be incorporated in microbiota analyses (Hayward et al. 2010; Albery et al. 2020). Applied to 
482 larger longitudinal data sets, our GLMM approach can allow researchers to directly estimate how

483 different aspects of host state, genotype and environment influence the structure of within-host

484 communities, and address many outstanding questions about the evolutionary and ecological

485 causes and consequences of host-microbe interactions.

487 Full realisation of the role of microbiota communities in the ecology and evolution of wild 488 organisms depends on both identifying factors with important effects on global microbiota 489 composition and on being able to test whether and how individual taxa or taxonomic groups

490 underpin those effects. Our GLMM approach readily lends itself to addressing both questions.

491 We have illustrated how the approach can be used to identify ASVs involved in community level 492 shifts with age identified in the random effects structure of the 2013 models (Figure 4), and to 493 further decompose the contribution of different taxonomic levels to community level effects 494 (ESM 1.4.3; Table S3). For example, our analysis highlights that analysis at the phylum level 495 could provide a misleading view of compositional shifts associated with age of Soay sheep, and 496 that there is substantial variation at the family level within each phylum (Figure S4). This 497 analysis approach should represent similar insights to linear discriminant analysis (LDA) used in 498 approaches such as LefSE (Segata et al. 2011), with the advantage of extraction of this 499 information for multiple factors of interest rather than requirement of a priori knowledge of 500 effects of interest to run differential abundance analysis. Our approach could be further 501 developed beyond the nested taxonomic levels used here to identify taxonomic levels associated 502 with the greatest variance (ESM 1.4.3) to explicitly include the microbiota phylogeny within the 503 GLMMs, allowing environment and host effects on community composition to be estimated 504 accounting more accurately for phylogenetic distances between ASVs (Hadfield \& Nakagawa 505 2010) in a similar manner to UNIFRAC clustering approaches (Lozupone et al. 2006). A

506 GLMM-based approach capable of simultaneously dissecting the contributions of host

507 environment, state and genetics alongside microbial phylogeny to variation in microbial

508 community structure seems to us to represent a powerful step towards robustly address emerging 509 questions surrounding the role of the microbiome in ecology and evolution. However, we note 510 however that there are computational challenges associated with incorporating more complex 511 covariance structures into our GLMMs, and provide some discussion of this issue in the 512 supplementary methods with a worked example of an alternate approach (ESM Section 2). 
514 Beyond applications to microbiota community analyses, approaches outlined in this manuscript

515 are applicable more broadly to different types of metataxonomic data being collected across

516 myriad systems and research disciplines. For example, there has been great interest in describing

517 the dynamics of the parasite community as an ecosystem and understanding its influence on host

518 health (Pedersen \& Fenton 2007; Graham 2008; Rynkiewicz et al. 2015). A growing number of

519 studies apply meta-barcoding to faecal samples to estimate the community structure of gastro-

520 intestinal parasite communities (Avramenko et al. 2015; Aivelo \& Medlar 2017), and our

521 GLMM approach could readily applied to such data sets to dissect the drivers of variation in

522 parasite community composition. Another area of interest within ecology and evolution is using

523 meta-barcoding of faecal samples to estimate diet composition and its relationship to host

524 phenotypes. Bayesian mixed model approaches have also recently been applied to analysis of

525 presence and absence of Cyanistes caeruleus (blue tit) diet components and align conceptually

526 with approaches presented in this manuscript (Shutt et al. 2020). GLMM approaches to

527 metabarcoding data maintain key similarities to other multivariate community ecology

528 approaches to abundance data (Wang et al. 2012) while integrating benefits of ecological and

529 evolutionary approaches to quantifying phenotypic variation. We therefore suggest that

530 approaches presented in this manuscript can be applied across a range of systems and data types

531 for powerful and flexible understanding of complex drivers of community dynamics.

\section{Acknowledgements}

534 This work was funded by a large NERC grant (NE/R016801/1), and the long-term study on St

535 Kilda was funded principally by responsive mode grants from NERC. We thank Adam Hayward and Jill Pilkington for sample collection, the National Trust for Scotland for support of our work

537 on St Kilda, and QinetiQ and Kilda Cruises for logistical support. We also thank the Ecology 538 Within Team for input in the analysis and manuscript and Josephine Pemberton for support and management of the field project. Figure 1 was created with Biorender.com. Photos on which sheep icons in Figure 1 are based by Hannah Vallin \& Martin Stoffel.

Author contributions.

LM and ARS conceived and developed the statistical methods; LM, ARS, AF, HL conducted the analyses; AF, AI, KAW, KW and DHN oversaw and undertook sample collection and laboratory work; ARS wrote the first draft of the manuscript and all authors contributed to writing of the final manuscript.

Data Accessibility

550 Sequences and metadata on which analysis is based can be found on the European Nucleotide

551 Archive (ENA): http://www.ebi.ac.uk/ena/data/view/PRJEB39322 


\section{References}

553

554

555

556

557

558

559

560

561

562

563

564

565

566

567

568

569

570

571

572

573

574

575

576

577

578

579

580

581

582

583

584

585

586

587

588

589

Aitchison, J. (1982). The Statistical Analysis of Compositional Data. Journal of the Royal Statistical Society: Series B (Methodological), 44, 139-160.

Aivelo, T. \& Medlar, A. (2017). Opportunities and challenges in metabarcoding approaches for helminth community identification in wild mammals. Parasitology, 145, 608-621.

Alberdi, A., Aizpurua, O., Bohmann, K., Zepeda-Mendoza, M.L. \& Gilbert, M.T.P. (2016). Do Vertebrate Gut Metagenomes Confer Rapid Ecological Adaptation? Trends in Ecology \& Evolution, 31, 689-699.

Albery, G.F., Becker, D.J., Kenyon, F., Nussey, D.H. \& Pemberton, J.M. (2019). The fine-scale landscape of immunity and parasitism in a wild ungulate population. Integrative and Comparative Biology, 16, e2003538.

Albery, G.F., Kirkpatrick, L., Firth, J.A. \& Bansal, S. (2020). Unifying spatial and social network analysis in disease ecology (D. Farine, Ed.). Journal of Animal Ecology, 30, 2009-18.

Amato, K.R., Leigh, S.R., Kent, A., Mackie, R.I., Yeoman, C.J., Stumpf, R.M., Wilson, B.A., Nelson, K.E., White, B.A. \& Garber, P.A. (2015). The gut microbiota appears to compensate for seasonal diet variation in the wild black howler monkey (Alouatta pigra). Microbial ecology, 69, 434-443.

Avramenko, R.W., Redman, E.M., Lewis, R., Yazwinski, T.A., Wasmuth, J.D. \& Gilleard, J.S. (2015). Exploring the Gastrointestinal 'Nemabiome': Deep Amplicon Sequencing to Quantify the Species Composition of Parasitic Nematode Communities (E. Serrano Ferron, Ed.). PLoS One, 10, e0143559-18.

Bolker, B.M., Brooks, M.E., Clark, C.J., Geange, S.W., Poulsen, J.R., Stevens, M.H.H. \& White, J.-S.S. (2009). Generalized linear mixed models: a practical guide for ecology and evolution. Trends in Ecology \& Evolution, 24, 127-135.

Callahan, B.J., McMurdie, P.J., Rosen, M.J., Han, A.W., Johnson, A.J.A. \& Holmes, S.P. (2016). DADA2: High-resolution sample inference from Illumina amplicon data. Nature methods, 13, 581583.

Carding, S., Verbeke, K., Vipond, D.T., Corfe, B.M. \& Owen, L.J. (2015). Dysbiosis of the gut microbiota in disease. Microbial Ecology in Health and Disease, 26, 1-10.

Clemente, J.C., Ursell, L.K., Parfrey, L.W. \& Knight, R. (2012). The Impact of the Gut Microbiota on Human Health: An Integrative View. Cell, 148, 1258-1270.

Consortium, T.I.S. (2009). Common polygenic variation contributes to risk of schizophrenia and bipolar disorder. Nature, 460, 748-752.

Cox-Foster, D.L., Conlan, S., Holmes, E.C., Palacios, G., Evans, J.D., Moran, N.A., Quan, P.-L., Briese, T., Hornig, M., Geiser, D.M., Martinson, V., vanEngelsdorp, D., Kalkstein, A.L., Drysdale, A., Hui, J., Zhai, J., Cui, L., Hutchison, S.K., Simons, J.F., Egholm, M., Pettis, J.S. \& Lipkin, W.I. (2007). A metagenomic survey of microbes in honey bee colony collapse disorder. Science, 318, 283-287.

Desbonnet, L., Clarke, G., Shanahan, F., Dinan, T.G. \& Cryan, J.F. (2014). Microbiota is essential for social development in the mouse. Molecular psychiatry, 19, 146-148. 
Foster, K.R., Schluter, J., Coyte, K.Z. \& Rakoff-Nahoum, S. (2017). The evolution of the host microbiome as an ecosystem on a leash. Nature, $\mathbf{5 4 8 ,} 43-51$.

Froy, H., Borger, L., Regan, C.E., Morris, A., Morris, S., Pilkington, J.G., Crawley, M.J., Clutton Brock, T.H., Pemberton, J.M. \& Nussey, D.H. (2018). Declining home range area predicts reduced late-life survival in two wild ungulate populations. Ecology letters, 21, 1001-1009.

Gelman, A. \& Hill, J. (2006). Data Analysis Using Regression and Multilevel/Hierarchical Models. Cambridge University Press.

597 Goldstein, D.B. (2009). Common Genetic Variation and Human Traits. N. Engl. J. Med., 1696-1703.

598 Graham, A.L. (2008). Ecological rules governing helminth-microparasite coinfection. Proceedings of the National Academy of Sciences of the United States of America, 105, 566-570.

600 Hadfield, J.D. (2010). MCMC methods for multi-response generalized linear mixed models: The MCMCglmm R Package. Journal of Statistical Software, 33, 1-22.

Hadfield, J.D. \& Nakagawa, S. (2010). General quantitative genetic methods for comparative biology: phylogenies, taxonomies and multi-trait models for continuous and categorical characters. Journal of Evolutionary Biology, 23, 494-508.

Hayward, A.D., Garnier, R., Watt, K.A., Pilkington, J.G., Grenfell, B.T., Matthews, J.B., Pemberton, J.M., Nussey, D.H. \& Graham, A.L. (2014). Heritable, heterogeneous, and costly resistance of sheep against nematodes and potential feedbacks to epidemiological dynamics. American Naturalist, 184, S58-S76.

Hayward, A.D., Pilkington, J.G., Pemberton, J.M. \& Kruuk, L.E.B. (2010). Maternal effects and early-life performance are associated with parasite resistance across life in free-living Soay sheep.

612 J, P., Glendinning, L., Wisedchanwet, T. \& Watson, M. (2018). The Madness of Microbiome: Attempting 613 To Find Consensus 'Best Practice' for 16S Microbiome Studies. Applied and environmental 614 microbiology, 84, e02627-17.

615 Jackson, M.M., Turner, M.G., Pearson, S.M. \& Ives, A.R. (2012). Seeing the forest and the trees: 616 multilevel models reveal both species and community patterns. Ecosphere, 3, 79.

617 Koskella, B., Hall, L.J. \& Metcalf, C.J.E. (2017). The microbiome beyond the horizon of ecological and 618 evolutionary theory. Nature Ecology \& Evolution, 1, 1606-1615.

619 Kruuk, L.E.B. \& Hadfield, J.D. (2007). How to separate genetic and environmental causes of similarity

Ley, R.E., Hamady, M., Lozupone, C., Turnbaugh, P.J., Ramey, R.R., Bircher, J.S., Schlegel, M.L., Tucker, T.A., Schrenzel, M.D., Knight, R. \& Gordon, J.I. (2008). Evolution of mammals and their gut microbes. Science, 320, 1647-1651.

Loh, P.-R., Bhatia, G., Gusev, A., Finucane, H.K., Bulik-Sullivan, B.K., Pollack, S.J., de Candia, T.R., Lee, S.H., Wray, N.R., Kendler, K.S., O'Donovan, M.C., Neale, B.M., Patterson, N. \& Price, A.L. (2015). Contrasting genetic architectures of schizophrenia and other complex diseases using fast variance-components analysis. Nature Genetics, 1-11. 
Lozupone, C., Hamady, M. \& Knight, R. (2006). UniFrac - An online tool for comparing microbial community diversity in a phylogenetic context. BMC Bioinformatics, 7.

Mandal, S., Van Treuren, W., White, R.A., Eggesbø, M., Knight, R. \& Peddada, S.D. (2015). Analysis of composition of microbiomes: a novel method for studying microbial composition. Microbial Ecology in Health and Disease, 26, 1-8.

Maurice, C.F., Knowles, S.C.L., Ladau, J., Pollard, K.S., Fenton, A., Pedersen, A.B. \& Turnbaugh, P.J. (2015). Marked seasonal variation in the wild mouse gut microbiota. ISME Journal, 9, 2423-2434.

McMurdie, P.J. (2018). Normalization of Microbiome Profiling Data. Microbiome Analysis, pp. 143-168. Methods and Protocols. Humana Press, New York, NY, New York, NY.

McMurdie, P.J. \& Holmes, S. (2014). Waste Not, Want Not: Why Rarefying Microbiome Data Is Inadmissible (A.C. McHardy, Ed.). PLoS computational biology, 10, e1003531.

Nakagawa, S. \& Schielzeth, H. (2013). A general and simple method for obtaining R2 from generalized

Niku, J., Hui, F.K.C., Taskinen, S. \& Warton, D.I. (2019). gllvm: Fast analysis of multivariate abundance data with generalized linear latent variable models in. Methods in Ecology and Evolution, 10, 21732182.

Orkin, J.D., Campos, F.A., Myers, M.S., Cheves Hernandez, S.E., Guadamuz, A. \& Melin, A.D. (2019). Seasonality of the gut microbiota of free-ranging white-faced capuchins in a tropical dry forest. Isme Journal, 13, 183-196.

Pedersen, A.B. \& Fenton, A. (2007). Emphasizing the ecology in parasite community ecology. Trends in Ecology \& Evolution, 22, 133-139.

Popkes, M. \& Valenzano, D.R. (2020). Microbiota-host interactions shape ageing dynamics. Philosophical Transactions of the Royal Society of London B: Biological Sciences, 375, $20190596-9$.

Ren, T., Boutin, S., Humphries, M.M., Dantzer, B., Gorrell, J.C., Coltman, D.W., McAdam, A.G. \& Wu, M. (2017). Seasonal, spatial, and maternal effects on gut microbiome in wild red squirrels. Microbiome, 5, 163-14.

657 Rynkiewicz, E.C., Pedersen, A.B. \& Fenton, A. (2015). An ecosystem approach to understanding and

Segata, N., Izaard, J., Waldron, L., Gevers, D., Miropolsky, L., Garrett, W.S. \& Huttenhower, C. (2011). managing within-host parasite community dynamics. Trends in Parasitology, 31, 212-221. model approach applied to faecal metabarcoding data. Molecular Ecology, 29, 1199-1213. 
664 Sudo, N., Chida, Y., Aiba, Y., Sonoda, J., Oyama, N., Yu, X.-N., Kubo, C. \& Koga, Y. (2004). Postnatal microbial colonization programs the hypothalamic-pituitary-adrenal system for stress response in mice. The Journal of physiology, 558, 263-275.

667 Vayssier-Taussat, M. (2014). Shifting the paradigm from pathogens to pathobiome: new concepts in the $668 \quad$ light of meta-omics. 1-7.

669 Visscher, P.M., Brown, M.A., McCarthy, M.I. \& Yang, J. (2012). REVIEW Five Years of GWAS

670 Discovery. The American Journal of Human Genetics, 90, 7-24.

671 Wahlström, A., Sayin, S.I., Marschall, H.-U. \& Bäckhed, F. (2016). Intestinal Crosstalk between Bile

672 Acids and Microbiota and Its Impact on Host Metabolism. Cell Metabolism, 24, 41-50.

673 Wang, Y., Naumann, U., Wright, S.T. \& Warton, D.I. (2012). mvabund- an Rpackage for model-based 674 analysis of multivariate abundance data. Methods in Ecology and Evolution, 3, 471-474.

675 Weiss, S.J., Xu, Z., Amir, A., Peddada, S., Bittinger, K., Gonzalez, A., Lozupone, C., Zaneveld, J.R., Vazquez-Baeza, Y., Birmingham, A. \& Knight, R. (2015). Effects of library size variance, sparsity, and compositionality on the analysis of microbiome data. PeerJ PrePrints, 3:e1157v1.

Weiss, S., Xu, Z.Z., Peddada, S., Amir, A., Bittinger, K., González, A., Lozupone, C., Zaneveld, J.R., Vázquez-Baeza, Y., Birmingham, A., Hyde, E.R. \& Knight, R. (2017). Normalization and microbial differential abundance strategies depend upon data characteristics. Microbiome, 5, 1-18.

681 Wilson, A.J. (2018). How should we interpret estimates of individual repeatability? Evolution Letters, 2, $682 \quad 4-8$.

683 Wilson, A.J., Réale, D., Clements, M.N., Morrissey, M.M., Postma, E., WALLING, C.A., Kruuk, L.E.B. 684 \& Nussey, D.H. (2010). An ecologist's guide to the animal model. Journal of Animal Ecology, 79, $685 \quad 13-26$.

686 Yuan, M.L., Dean, S.H., Longo, A.V., Rothermel, B.B., Tuberville, T.D. \& Zamudio, K.R. (2015). Kinship, inbreeding and fine-scale spatial structure influence gut microbiota in a hindgut-fermenting tortoise. Molecular Ecology, 24, 2521-2536. 